\title{
Influence of Hydrogen on Local Mechanical Properties of Pure Fe with Different Dislocation Densities Investigated by Electrochemical Nanoindentation
}

\author{
Kota TOMATSU, (1** Tomohiko OMURA, ${ }^{1)}$ Yoshitaka NISHIYAMA ${ }^{1)}$ and Yoshikazu TODAKA ${ }^{2)}$ \\ 1) Steel Research Laboratories, Technical Research \& Development Bureau, Nippon Steel \& Sumitomo Metal Corporation, 1-8 \\ Fuso-cho, Amagasaki-shi, Hyogo, 660-0891 Japan. \\ 2) Toyohashi University of Technology, 1-1 Hibarigaoka, Tempaku-cho, Toyohashi, Aichi, Japan.
}

(Received on April 7, 2016; accepted on August 1, 2016)

\begin{abstract}
In order to investigate influence of dislocation density on hydrogen embrittlement behavior, local mechanical properties of pure Fe with different dislocation densities were measured during hydrogen charging at various load duration times by electrochemical nanoindentation. For as-annealed samples and severely plastically deformed ones with low temperature annealing, hydrogen charging did not change nanohardness at any load duration times between $1 \mathrm{~s}$ and $10800 \mathrm{~s}$. On the other hand, for cold-rolled samples and severely plastically deformed ones without annealing, hydrogen charging caused softening, and the degree of the softening increased at longer load duration times. Consequently, it was found that hydrogen causes softening for samples with higher dislocation density at slower strain rates. The observed softening seems to be caused by increase in dislocation mobility or suppression of work-hardening due to hydrogen atoms trapped around dislocations.
\end{abstract}

KEY WORDS: nanoindentation; hydrogen embrittlement; dislocation density; iron.

\section{Introduction}

It is well known that high-strength steel is susceptible to hydrogen embrittlement (HE), where strength or toughness of steel is degraded by hydrogen. ${ }^{1,2)}$ One of reasons for higher HE susceptibility of high-strength steel is likely that high-strength steel, such as martensitic one, generally contains a large number of dislocations, and hydrogen interacts with these dislocations. Thus, in order to suppress $\mathrm{HE}$, elucidation of influence of dislocation density on HE behavior is critical.

Regarding the influence of dislocation density, M. Nagumo et al. observed microstructures of pure Fe strained during hydrogen charging, and found that hydrogen nucleates microvoids along deformation bands, where dislocation density is high. ${ }^{3)}$ On the basis of the observed results, they proposed that a linkage of these microvoids results in HE fracture. Y. Todaka et al. investigated HE susceptibility of ultra-low carbon steel with ultrafine grains and different dislocation densities by small punch tests in gaseous hydrogen. ${ }^{4)}$ The samples were made by severe plastic deformation with high-pressure torsion (HPT) and subsequent annealing. They reported that HE occurs for the higher dislocation density sample, whereas HE is suppressed for the lower dislocation density sample. Moreover, D.-H. Lee et al. investigated influence of hydrogen on mechanical properties of linepipe

\footnotetext{
* Corresponding author: E-mail: tomatsu.rk6.kohta@jp.nssmc.com DOI: http://dx.doi.org/10.2355/isijinternational.ISIJINT-2016-189
}

steel by nanoindentation with indenters with different sharpness. ${ }^{5)}$ They found that hydrogen charging causes hardening for blunt indenters, and softening for sharp indenters. It was proposed that the observed difference in influence of hydrogen on nanohardness originates from difference in density of dislocations generated under the indenter: for the sharper indenter, more dislocations are generated, and hydrogen's elastic shielding effect is enhanced.

In recent years, electrochemical nanoindentation (EC-NI), where indentations are performed during hydrogen charging in an electrochemical cell, has attracted much attention as an experimental method to explore hydrogen-dislocation interactions at a microscopic scale. ${ }^{6,7)}$ The authors also developed EC-NI apparatus, and improved it so that indentations can be performed at extremely long load duration times, where HE is pronounced, by compensating thermal drift (TD) of the indenter. ${ }^{8)}$ Furthermore, the developed EC-NI apparatus was applied to face-centered cubic (fcc) metal without strains, and the tendency was revealed that hydrogen causes softening inside grains at slower strain rates. It was considered that the observed softening is caused by enhancement in dislocation mobility or suppression of work-hardening by hydrogen atoms around dislocations.

More insights can be gained on how dislocation density is associated with HE behavior by using EC-NI for bodycentered cubic metal. On the other hand, use of samples with alloy elements or precipitates, such as practical steel, seems to complicate interpretation of observed results. Thus, in the present study, pure Fe with different dislocation den- 
sities were made, and influence of hydrogen on their local mechanical properties was measured by EC-NI.

\section{Experimental}

Following the steps shown in Fig. 1, pure Fe was subjected to cold rolling and severe plastic deformation, and samples with different dislocation densities were made: as-annealed (AA), cold-rolled (CR), and severely plastically deformed samples (SPD). The annealing was performed in vacuum at $1273 \mathrm{~K}$ for more than $1800 \mathrm{~s}$. A reduction rate of the cold rolling was set to $80 \%$. On the other hand, the severe plastic deformation was performed by HPT process ${ }^{9}{ }^{9}$ where disc samples with a diameter of $10 \mathrm{~mm}$ and a thickness $t$ of $0.85 \mathrm{~mm}$ were torsionally deformed under compressive pressure of $5 \mathrm{GPa}$ at room temperature. The number of turns $N$ was set to five. After the HPT process, $t$ was decreased to $0.60 \mathrm{~mm}$. A sample with almost the same grain size as, but lower dislocation density than SPD, was also tried to be made by annealing SPD at $473 \mathrm{~K}$ for 3600 $\mathrm{s}(\mathrm{SPD}+\mathrm{A})$ as in Ref. 4). Purity of Fe is shown in Table 1. Commercial pure Fe (Nilaco, 99.99\%) with 6 ppm C was used for AA and CR, and pure Fe with 11 ppm C for SPD and SPD $+\mathrm{A}$.

For measuring areas, where indenters are pressed by EC-NI, rolling planes were selected for $\mathrm{CR}$, and areas near the disc center at $r \approx 1 \mathrm{~mm}$ ( $r$ is a distance from the disk center) for SPD and SPD+A. Equivalent strain of the measuring area of CR is 1.9 , and that of SPD calculated from Refs. 10) and 11) $(N=5, t=0.60 \mathrm{~mm}$, and $r=1 \mathrm{~mm})$ is 4.57. Nanohardness of the measuring area in the air were 2.5 GPa, 3.2 GPa, 3.7 GPa, and 3.6 GPa for AA, CR, SPD, and SPD $+\mathrm{A}$, respectively. For SPD, nanohardness was identical in the areas of $r \geq 0.5 \mathrm{~mm}$. The nanohardness in the air was obtained at load duration time $\Delta t$ of $5 \mathrm{~s}$ with the same experimental conditions as for the EC-NI measure-

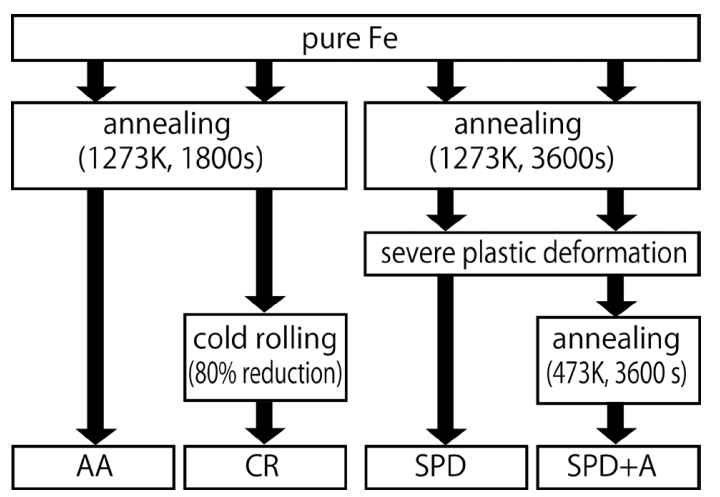

Fig. 1. Preparation steps for samples with different dislocation densities. ments described below. In order to obtain flat and clean surfaces, the sample surface including the measuring area was mechanically polished and finished by electrochemical polishing.

A schematic of experimental setup of the EC-NI measurements is illustrated in Fig. 2. A diamond cube-corner indenter was pressed into the sample surfaces under load control in electrolyte with and without hydrogen charging at room temperature. In order to investigate strain rate dependence, $\Delta t$ was changed between $0.1 \mathrm{~s}$ and $10800 \mathrm{~s}$ at common maximum displacement of about $200 \mathrm{~nm}$. For $\Delta t<5 \mathrm{~s}$, nanohardness was obtained by analyzing recorded load-displacement curves by an Oliver-Pharr method. ${ }^{12)}$ On the other hand, for $\Delta t$ typically more than $600 \mathrm{~s}$, TD of the indenter causes inaccuracy in the recorded load-displacement curves. Therefore, for $\Delta t \geq 5 \mathrm{~s}$, load-displacement curves and load-nanohardness ones were indirectly calculated from Young's modulus and load-contact stiffness relations recorded by modulating the load. The details of the TD compensation method have been described elsewhere. ${ }^{8)}$ Frequency and amplitude of the load modulation were set to $200 \mathrm{~Hz}$ and $1.25 \%-3 \%$ of the applied load, respectively. Young's modulus, which is necessary for the TD compensation, was separately obtained from indentations at $\Delta t=5 \mathrm{~s}$, where TD is negligible.

Hydrogen charging was performed under current control, and cathodic current density was set to $2.5 \mathrm{~A} / \mathrm{m}^{2}$. For electrolyte and a counter electrode, borate buffer solution $(\mathrm{pH} 8.62)$ and a $\mathrm{Pt}$ wire were used, respectively. It was confirmed that, in the present hydrogen charging conditions, no corrosion occurs at the sample surfaces during the measurements. Prior to the indentations during the hydrogen charging, precharge was performed for more than $1200 \mathrm{~s}$ at the same cathodic current density (i.e., $2.5 \mathrm{~A} / \mathrm{m}^{2}$ ) to saturate hydrogen concentration in the sample subsurface. It was confirmed by thermal desorption analysis that the present hydrogen charging condition is mild, and about $0.1 \mathrm{ppm}$ of diffusive hydrogen is charged for AA.

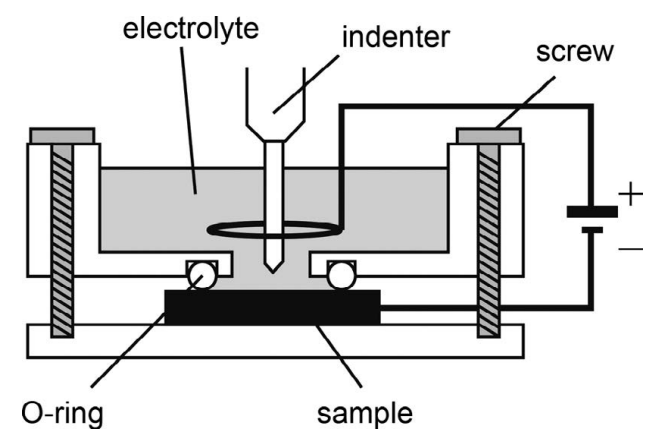

Fig. 2. Schematic of experimental setup of EC-NI measurements.

Table 1. Chemical composition of pure Fe used in the present study. The unit is mass $\%$.

\begin{tabular}{cccccccccccccccccccccc}
\hline Samples & $\mathrm{Fe}$ & $\mathrm{C}$ & $\mathrm{Si}$ & $\mathrm{Mn}$ & $\mathrm{P}$ & $\mathrm{S}$ & $\mathrm{Cr}$ & $\mathrm{N}$ & $\mathrm{O}$ & $\mathrm{Al}$ & $\mathrm{Cu}$ & $\mathrm{Ti}$ \\
\hline $\begin{array}{c}\text { AA } \\
\text { CR }\end{array}$ & Bal. & 0.0006 & 0.002 & $<0.001$ & 0.0007 & 0.0003 & $<0.001$ & 0.0008 & 0.0171 & NA & NA & NA \\
\hline $\begin{array}{c}\text { SPD } \\
\text { SPD +A }\end{array}$ & Bal. & 0.0011 & $<0.003$ & $<0.003$ & $<0.002$ & $<0.0003$ & $<0.003$ & 0.0008 & 0.0014 & 0.030. & $<0.003$ & $<0.002$
\end{tabular}

NA: Not analyzed 
Textures and degree of deformation of each sample were confirmed by measuring inverse pole figure (IPF) images and Kernel average misorientation (KAM) ones of electron back-scattering diffraction (EBSD). For SPD and SPD+A, the images were obtained at the positions of $r \geq 0.5 \mathrm{~mm}$. The dislocation density was also confirmed by analyzing (110), (211), and (220) reflections of X-ray diffraction (XRD) by a Williamson-Hall method. ${ }^{13)}$ Analysis area of $\mathrm{XRD}$ is about $20 \mathrm{~mm}$ in diameter.

\section{Results}

\subsection{Microstructure and Dislocation Density}

The sample AA was composed of equiaxed grains, and an average grain size was about $300 \mu \mathrm{m}$. IPF and KAM images of CR, SPD, and SPD + A are shown in Figs. 3 to 5, respectively. Frequency distribution of the KAM value is also shown for SPD and SPD+A. The blank (black) areas near the grain boundary in the KAM images correspond to the areas where Kikuchi bands of EBSD are ambiguous. The KAM data in these areas were excluded to obtain the frequency distribution. For CR, large non-equiaxed grains and ultrafine ones with a diameter of about $1 \mu \mathrm{m}$ were formed (Fig. 3). High KAM areas appeared both in the nonequiaxed grains and the ultrafine ones. On the other hand, SPD and SPD+A were fully composed of ultrafine grains (Figs. 4 and 5). An average grain size was almost the same between SPD and SPD+A: $0.41 \mu \mathrm{m}$ for SPD and $0.53 \mu \mathrm{m}$
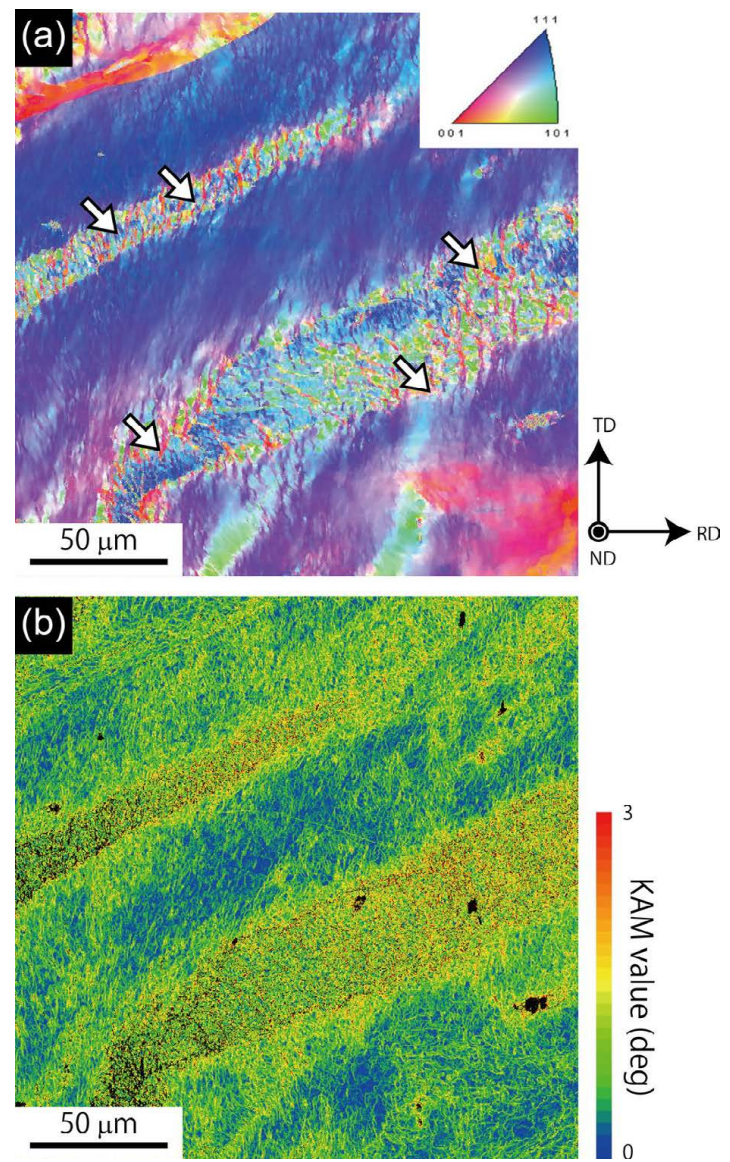

Fig. 3. (a) IPF and (b) KAM images of CR. The arrows represent the areas where ultrafine grains are formed. (Online version in color.) for SPD+A. High KAM areas were fewer for SPD+A than for SPD, indicating that dislocation density is decreased and strain is released by the annealing at $473 \mathrm{~K}$.

In Table 2, dislocation density of each sample obtained by XRD is shown. The sample with the highest dislocation density was SPD, and the dislocation density was the order of $10^{15} \mathrm{~m}^{-2}$. In contrast, AA contained very few dislocations, and the dislocation density was the order of $10^{12}$ $\mathrm{m}^{-2}$. In accordance with the KAM images in Figs. 4 and 5, dislocation density of SPD + A was smaller than that of SPD. The dislocation densities of SPD + A was slightly smaller than that of $\mathrm{CR}$, and the order of $10^{14} \mathrm{~m}^{-2}$.

\subsection{Influence of Hydrogen on Local Mechanical Prop- erty}

The indenter was pressed into interiors of large equiaxes grains for $\mathrm{AA}$, and interiors of large non-equiaxes grains
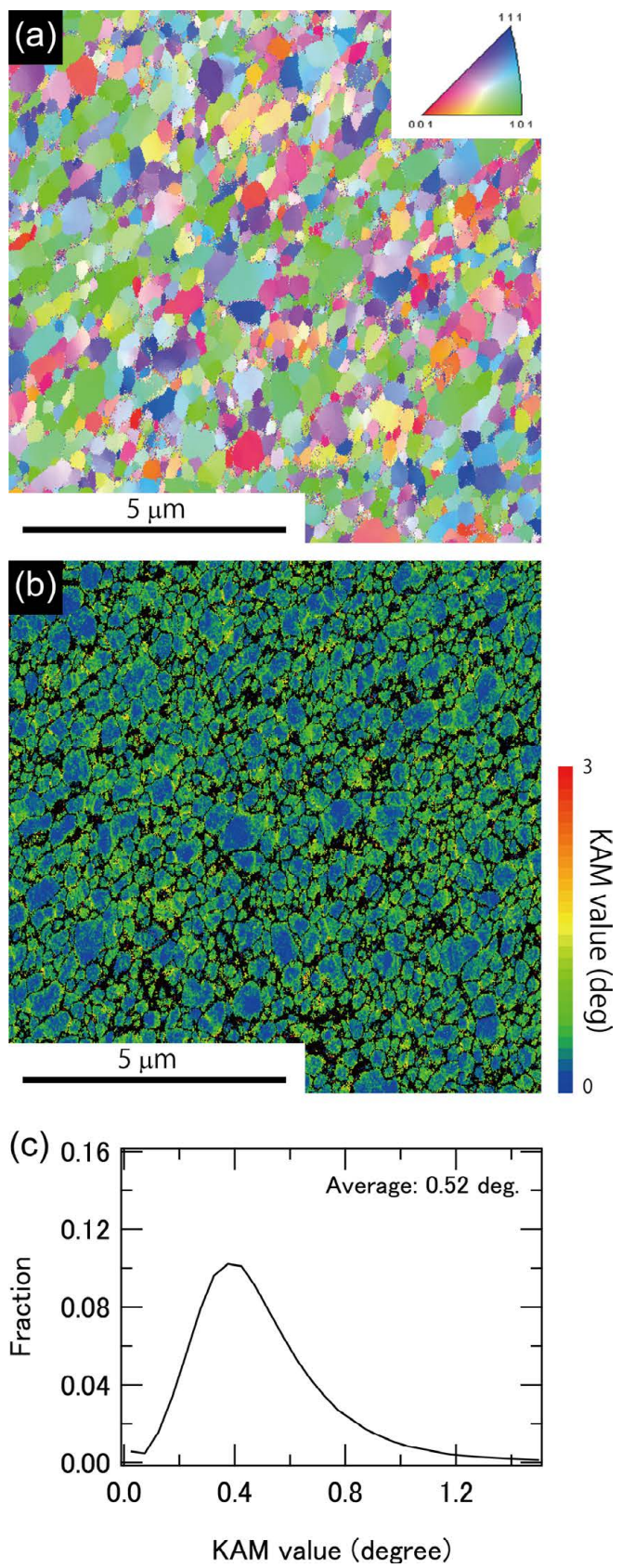

Fig. 4. (a) IPF image, (b) KAM image, and (c) frequency distribution of KAM value of SPD. (Online version in color.) 

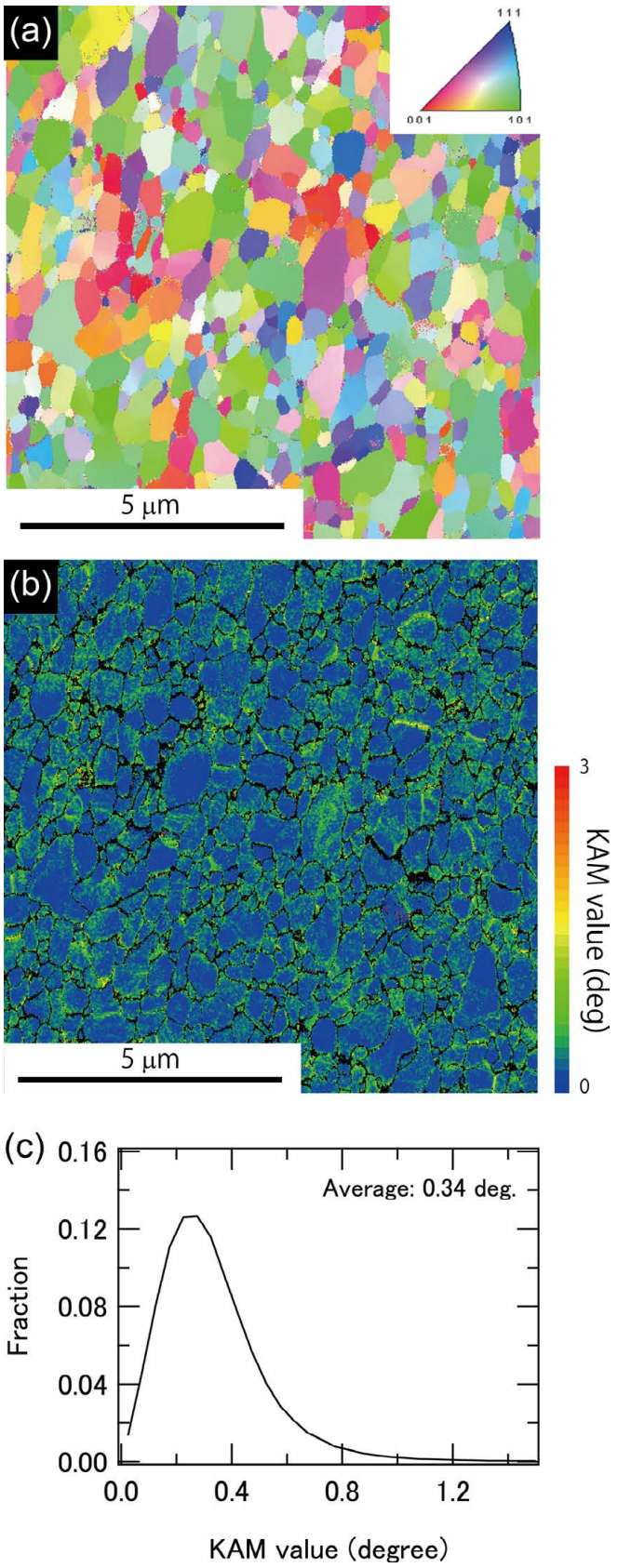

Fig. 5. (a) IPF image, (b) KAM image, and (c) frequency distribution of KAM value of SPD+A. (Online version in color.)

Table 2. Dislocation densities of each sample measured by XRD.

\begin{tabular}{ccccc}
\hline & AA & CR & SPD & SPD + A \\
\hline Dislocation density $\left(\mathrm{m}^{-2}\right)$ & $1.7 \times 10^{12}$ & $5.8 \times 10^{14}$ & $1.5 \times 10^{15}$ & $4.0 \times 10^{14}$ \\
\hline
\end{tabular}

for CR. On the other hand, the indenter was pressed into multiple grains simultaneously for SPD and SPD $+\mathrm{A}$. It is noted that the grains of SPD and SPD $+\mathrm{A}$ are ultrafine as shown in Figs. 4 and 5, and an imprint formed on the surface (about $1 \mu \mathrm{m}^{2}$ ) is inevitably larger than the grain size. For $\mathrm{AA}$ and $\mathrm{CR}$, data at the same $\Delta t$ with and without hydrogen charging were obtained from the identical grain to eliminate influence of crystalline orientation.

Young's modulus obtained at $\Delta t=5 \mathrm{~s}$, where TD effect is negligible, was not changed by hydrogen charging for all the samples. By using these Young's moduli, load-displacement curves without the TD effect were calculated for $\Delta t \geq 5 \mathrm{~s}$. In Fig. 6, load-displacement curves at typical $\Delta t$ obtained with and without hydrogen charging are shown. The curves at $\Delta t \leq 600 \mathrm{~s}$ are an average of five data, and those at $\Delta t>$ $600 \mathrm{~s}$ are an average of three data. It is noted that unloading segments of the load-displacement curves at $\Delta t=600$ $\mathrm{s}$ and $3600 \mathrm{~s}$ are not plotted because the TD compensation was not applied during the unloading. ${ }^{8)}$ It is also noted that, in the TD compensation, larger load amplitude was used for SPD+A than for the other samples, resulting in smaller signal-to-noise ratio for the load-displacement curves of $\mathrm{SPD}+\mathrm{A}$. For AA and SPD $+\mathrm{A}$, maximum displacement was not changed by the hydrogen charging at any $\Delta t$, indicating that nanohardness is not changed. On the other hand, for $\mathrm{CR}$ and SPD, maximum displacement was not changed at $\Delta t=1 \mathrm{~s}$, but increased at $\Delta t \geq 600 \mathrm{~s}$. The increase in the maximum displacement indicates that softening occurs.

In Fig. 7, relations between $\Delta t$ and the change in nanohardness by hydrogen charging corresponding to the data in Fig. 6 are shown. Nanohardness at different $\Delta t$ was obtained from different grains even for $\mathrm{AA}$ and $\mathrm{CR}$. In order to eliminate influence of crystalline orientation, the change in $H_{n}$ was represented by the rate $\Delta H_{n}$ given by the following equation:

$$
\Delta H_{n}=\left(\frac{H_{n}^{w i t h H}}{H_{n}^{w / o H}}-1\right) \times 100[\%],
$$

where $H_{n}^{\text {withH }}$ and $H_{n}^{w / o H}$ are nanohardness with and without hydrogen charging at the same load, respectively. For AA and $\mathrm{SPD}+\mathrm{A}, \Delta H_{n}$ was around zero at any $\Delta t$ between 0.1 $\mathrm{s}$ and $10800 \mathrm{~s}$. On the other hand, for CR and SPD, $\Delta H_{n}$ was decreased with increase in $\Delta t$, and the tendency was found that larger softening is caused at longer $\Delta t$. Degree of the softening was larger for SPD than CR, and $\Delta H_{n}$ at $\Delta t=3600 \mathrm{~s}$ was about $-20 \%$ and $-10 \%$ for SPD and CR, respectively.

\section{Discussion}

It was revealed by the EC-NI measurements that hydrogen charging does not affect nanohardness for AA and $\mathrm{SPD}+\mathrm{A}$, but causes softening for $\mathrm{CR}$ and SPD at longer $\Delta t$ (Fig. 7). On the other hand, primary difference between SPD and SPD $+\mathrm{A}$ is dislocation density, and the grain size is the almost same between these two samples. This means that grain boundaries, which are likely to interact with hydrogen, do not contribute to the softening so much. Thus, the present EC-NI results indicate that softening is caused by hydrogen for samples with higher dislocation density at slower strain rates. In order to clarify this point, relation between $\Delta H_{n}$ at $\Delta t=3600 \mathrm{~s}$ and the dislocation density obtained by XRD (Table 2) is plotted in Fig. 8. $\Delta H_{n}$ at $\Delta t=3600 \mathrm{~s}$ was obtained by fitting the data in Fig. 7 to a straight line in logarithmic scale,

$$
\Delta H_{n}=A \log \Delta t+B,
$$

where $A$ and $B$ are fitting parameters. It is confirmed from Fig. 8 that hydrogen decreases nanohardness for samples with dislocation density more than about $5 \times 10^{14} \mathrm{~m}^{-2}$. The present EC-NI results are also qualitatively consistent 
(a)

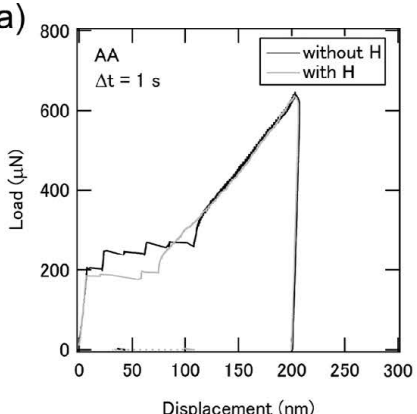

(b)

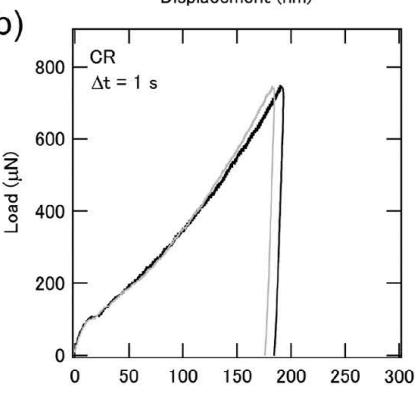

(c)

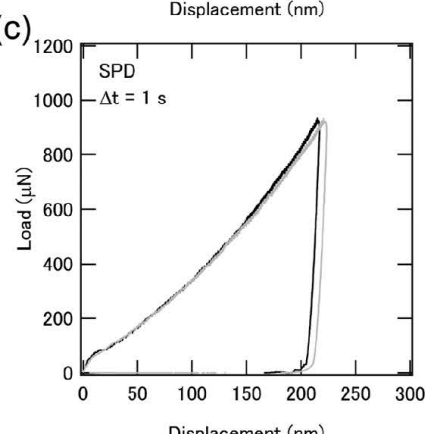

(d)

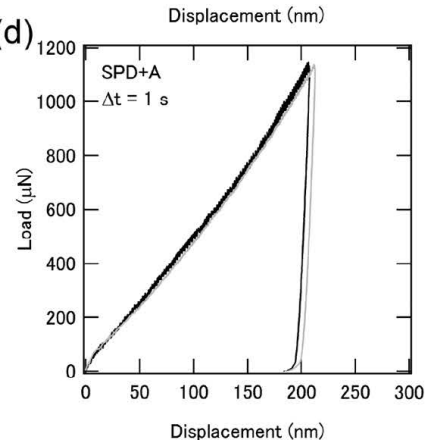

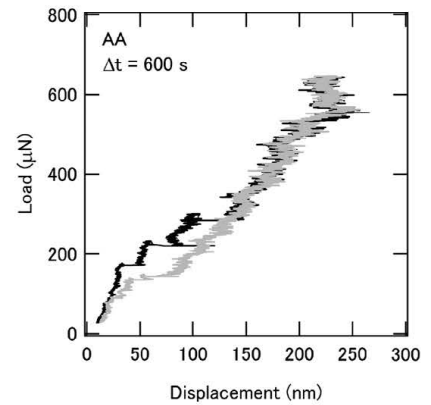
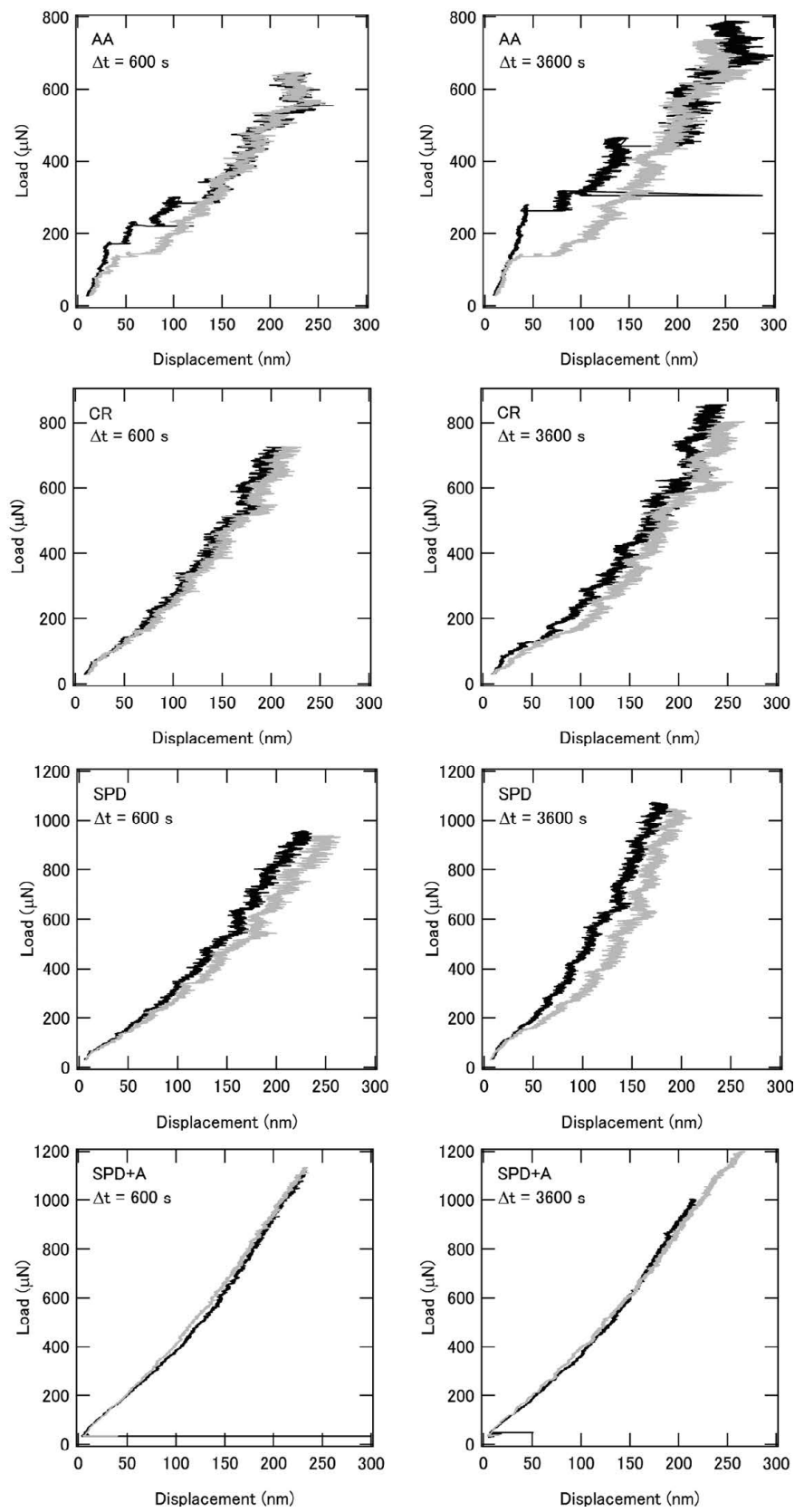

Fig. 6. Load-displacement curves with and without hydrogen charging at different $\Delta t$ of (a) AA, (b) CR, (c) SPD, and (d) SPD +A.

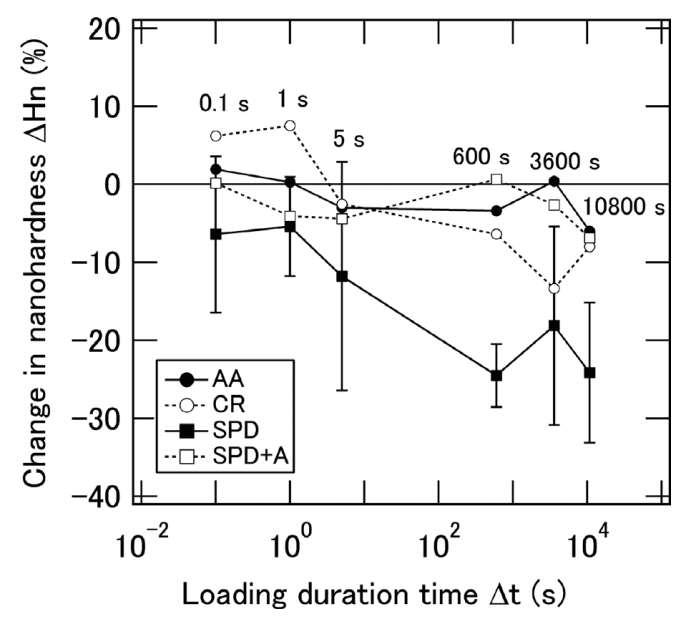

Fig. 7. Relation between $\Delta t$ and $\Delta H_{n}$. Error bars are shown for SPD as a representative.

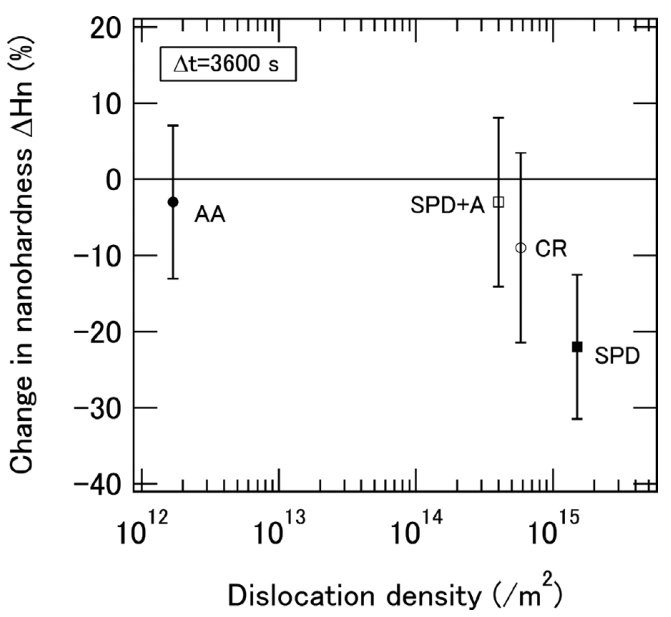

Fig. 8. Relation between dislocation densities obtained by XRD and $\Delta H_{n}$ at $\Delta t=3600 \mathrm{~s}$. 
with the nanoindentation results by D.-H. Lee et al., where softening is caused by hydrogen for sharper indenters generating more dislocations. ${ }^{5}$ )

Regarding the mechanism of the hydrogen-induced softening at slow strain rates in the EC-NI measurements of fcc metals, the authors have proposed two models: (i) increase in dislocation mobility (e.g., decrease in energy barrier for kink-pair nucleation or kink migration in screw dislocations) and (ii) suppression of work-hardening. ${ }^{8)}$ The latter is caused, for example, by hydrogen-enhanced cross-slip ${ }^{14)}$ or reduction in repulsive interaction among dislocations due to shielding of elastic field by hydrogen. ${ }^{15,16)}$ In both models, hydrogen atoms trapped around the dislocations play a roll. Therefore, softening is pronounced at slower strain rates, where the dislocations move slowly and more hydrogen atoms can accumulate around the dislocations. ${ }^{17)}$ One might think that contribution of dislocation mobility change to $H_{n}$ is trivial because dislocation intersections frequently occur in highly strained volume under the indenter, which likely dominates the deformation resistance of the sample. ${ }^{18)}$ On the other hand, it is worth noting that interstitial solute atoms (e.g., carbon) diffusing in a lattice at elevated temperatures can decrease dislocation mobility, and increases deformation resistance in tensile tests even in high strain region (dynamic strain aging). ${ }^{19)}$ This suggests that the increase in dislocation mobility by hydrogen can decrease the deformation resistance in high strain region and hence $H_{n}$.

Y. Koizumi et al. suggested by internal friction measurements and transmission electron microscopy that ultrafine grains of aluminum obtained by accumulative rolling bonding contain a large number of mobile dislocations. ${ }^{20)}$ Given that SPD or CR similarly contains a large number of mobile dislocations, the observed hydrogen-induced softening of these samples is basically understood by the same models as for the fcc metals. Relation between dislocation velocity $v_{d}$ and a strain rate $\dot{\gamma}$ is given by the following equation,

$$
\dot{\gamma}=\rho b v_{d},
$$

where $\rho$ and $b$ are density of mobile dislocations and a Burgers vector, respectively. ${ }^{21)}$ Equation (3) means that, for samples with higher mobile dislocation density, the mobile dislocations move more slowly, and interact with hydrogen more strongly at a given strain rate. That is, increase in dislocation density is equivalent to decrease in strain rates. It seems that the softening for SPD or CR is also caused by increase in dislocation mobility or suppression of workhardening.

\section{Summary}

In order to investigate influence of dislocation density on HE behavior at a microscopic scale, local mechanical properties of pure Fe with different dislocation densities with and without hydrogen charging were measured by EC-NI. As a result, the following findings were obtained.

1. For $\mathrm{AA}$ and $\mathrm{SPD}+\mathrm{A}$, hydrogen charging did not change nanohardness at any $\Delta t$ between $0.1 \mathrm{~s}$ and 10800 s. On the other hand, for CR and SPD, hydrogen charging decreased nanohardness at longer $\Delta t$. The tendency was revealed that softening is caused by hydrogen for samples with higher dislocation density at slower strain rates.

2. The present results are consistent with the previous nanoindentation results by D.-H. Lee et al., where softening is caused by hydrogen for sharp indenters.

3. It is considered that, for the samples with higher dislocation density, dislocation moves slowly and more strongly interacts with hydrogen. The observed softening by hydrogen charging can be attributed to increase in dislocation mobility or suppression of work-hardening by hydrogen.

\section{Acknowledgements}

The authors would like to acknowledge fruitful discussions with Y. Sakiyama at Nippon Steel \& Sumitomo Metal Corporation, and H. Sato at Toyohashi University of Technology.

\section{REFERENCES}

1) R. A. Oriani: Ann. Rev. Mater. Sci., 8 (1978), 327.

2) M. Nagumo: Fundamentals of Hydrogen Embrittlement, Uchida Rokakuho Publishing, (2008), (in Japanese).

3) M. Nagumo and K. Miyamoto: J. Jpn. Inst. Met., 45 (1981), 1309 (in Japanese).

4) Y. Todaka, K. Morisato, M. Kumagai, Y. Matsumoto and M. Umemoto: Adv. Mater. Res., 89-91 (2010), 763.

5) D.-H. Lee, J.-A. Lee, M.-Y. Seok, U. B. Baek, S. H. Nahm and J. Jang: Int. J. Hydrog. Energy, 39 (2014), 1897.

6) A. Barnoush and H. Vehoff: Acta Mater., 58 (2010), 5274

7) A. Barnoush, J. Dake, N. Kheradmand and H. Vehoff: Intermetallics, 18 (2010), 1385.

8) K. Tomatsu, K. Miyata and T. Omura: ISIJ Int., 56 (2016), 418.

9) R. Z. Valiev, R. K. Islamgaliev and I. V. Alexandrov: Prog. Mater. Sci., 45 (2000), 103.

10) A. P. Zhilyaev and T. G. Langdon: Prog. Mater. Sci., 53 (2008), 893.

11) S. Onaka: J. Jpn. Inst. Met. Mater., 74 (2010), 165 (in Japanese).

12) W. C. Oliver and G. M. Pharr: J. Mater. Res., 7 (1992), 1564.

13) G. K. Williamson and W. H. Hall: Acta Metall., 1 (1953), 22.

14) K. Miyata: Metall. Mater. Trans. A, 34A (2003), 1249.

15) H. K. Birnbaum and P. Sofronis: Mater. Sci. Eng. A, 176 (1994), 191.

16) P. Sofronis and H. K. Birnbaum: J. Mech. Phys. Solids, 43 (1995), 49.

17) S. Taketomi, R. Matsumoto and N. Miyazaki: J. Mater. Res., 26 (2011), 1269.

18) M. Wen, L. Zhang, B. An, S. Fukuyama and K. Yokogawa: Phys. Rev. B, 80 (2009), 094113.

19) B. J. Brindley and J. T. Barnby: Acta Metall., 16 (1968), 41.

20) Y. Koizumi, M. Ueyama, N. Tsuji, Y. Minamino and K. Ota: J. Alloy. Compd., 355 (2003), 47.

21) J. P. Hirth and J. Lothe: Theory of Dislocations, John Wiley \& Sons, USA, (1982). 